\title{
THE ASSOCIATION OF CLINICAL PATHOLOGISTS
}

The 64th general meeting was held at the University College of South Wales and Monmouthshire, Cardiff, on April 7, 8, and 9, 1960.

Abstracts of papers given follow.

\section{Effect of Adrenalin Infusion on the Plasma Concentrations of Antihaemophilic Globulin in Normal Subjects and Haemophiliacs}

G. I. C. INGRAM (St. Thomas's Hospital, London) said that experiences in two haemophiliacs suggested that acute, severe bleeding was associated with a sudden rise in plasma antihaemophilic globulin (A.H.G.) activity. This seemed analogous to a previous observation that the clotting time in siliconecoated tubes shortened markedly after adrenalin infusions in normal subjects; and the effect of adrenalin on plasma A.H.G. activity was therefore investigated. Adrenalin (10-15 $\mu \mathrm{g} . / \mathrm{min}$.), isoprenalin, (3-5 $\mu \mathrm{g} . / \mathrm{min}$.$) , and noradrenalin (10-15 \mu \mathrm{g} . / \mathrm{min}$.) were infused for $10 \mathrm{~min}$. in six normal subjects; so far, on the average, A.H.G. activity had risen after each drug, but most with adrenalin, with considerable individual variation. In two haemophiliacs, a marked rise was noted in one patient after two adrenalin infusions, but a fall in the other patient ; in two severe haemophiliacs, there was no rise.

Other experiments suggested that the effect is not an artifact related to contact factors or to fibrinolytic activity, and does not appear to be related to a fall in the eosinophil count; a suggestive correlation has been observed between the A.H.G. rise and the rise in blood glucose concentration.

\section{Listerian Control of Staphylococcal Hospital Cross-infection}

S. D. EleK and P. C. Fleming (St. George's Hospital, London) said that Lister's carbolic spray was replaced by aseptic surgery, but staphylococcal wound infection is still a problem. The main reservoir of staphylococci is believed to be the nose, from which dust, fresh individuals, and wounds are infected. The risk of wound infection in hospitals is related to the degree of pollution of the environment.

Gould showed that in hospitals there is a detectable amount of penicillin floating in the air, leading to a selective exclusion of penicillin-sensitive staphylococci from the noses of personnel. The authors made a deliberate use of this observation to eliminate nasal carriage. A new experimental antibiotic, AB 1241. was selected as it fulfilled the following requirements: (1) It was active against all of 400 strains of Staph. pyogenes tested. (2) It was active in concentrations of 1 to $5 \mu \mathrm{g}$. per ml. and highly bactericidal at about the bacteriostatic level. (3) Resistance could not be induced in 12 strains by 20 serial passages through Szybalski gradient plates. The substance is odourless and non-irritant.

A maternity unit was chosen for the spraying experiment because of the known high incidence of nasal carriage in infants. Approximately $55 \%$ of daily nasal swabs taken from each of 58 newborn infants during their first 10 days of life grew pyogenic staphylococci. Daily spraying of $1 \mathrm{~g}$. of the drug was next carried out in one ward and nursery occupying about $15,000 \mathrm{cu}$. ft., using a commercial windowcleaning sprayer. This halved the nasal carriage but failed to abolish it. Increasing the concentration to $1 \mathrm{~g}$. four times a day led to further reduction for the next 20 babies. Thereafter a cumulative effect became manifest as the drug began to build up in the environment. No pyogenic staphylococci at all were isolated from the next 24 babies during their 10-day stay in hospital. No resistant strains emerged, and no hypersensitivity phenomena have been observed over the 20-week period. This appears to be a simple and effective means of eliminating nasal carriage in babies, and is put forward as a possible approach for the control of wound sepsis in hospitals.

\section{The Nature of Vaginal Discharge}

J. A. Boycotr (Taunton) reported that, of 561 specimens of vaginal discharge from women between the ages of 15 and 50, 418 contained little or no pus. The reaction, cytology, and flora of this non-purulent discharge make it probable that it is an exaggerated flow of the normal secretions of the vagina and cervix. One hundred and thirty-four out of 143 purulent discharges contained Trichomonas spp. with a mixed and inconstant bacterial flora. Nine purulent discharges appeared to be due to bacterial infection.

Candida spp. were found in one purulent and in 64 non-purulent discharges. It is suggested that these yeasts are not parasitic upon the vaginal epithelium but derive their nutrition from the secretions of the epithelium.

In children below 15 and in women over 50 the picture is different. The discharge in 14 out of 16 children and in 16 out of 18 older women appeared to be due to bacterial infection.

\section{Cross-infection in Maternity Hospitals}

Beryl D. Corner and William A. Gillespie (Bristol) also spoke of cross-infection in maternity hospitals, and showed that the spread of staphylococcal infection was quite acute once there was an accumulation of resistant strains. They found that the ordinary accepted methods of interruption of spread were disappointing in the long run, and that the infection arose from adult carriers and was spread superficially by nose and skin, particularly the umbilicus. By the continuous use of hexachlorophane powder and by the instillation of hibitane and neomycin in the noses of mothers and babies there was 
a marked reduction in the staphylococcal rate in both mothers and babies, particularly in the follow-up clinics in the incidence of breast abscesses and sticky eyes in the children. A word of warning was, however, sounded in that it was evident in the most recent survey that Monilia were now taking a firmer hold in the wards, and in the last bacterial survey there were 25 cases of spots due to staphylococci, three streptococcal abscesses, and 72 cases of monilial infection. When asked whether the nose was still the main source of infection, they felt that as regards the babies the umbilicus was probably more important, as it became colonized rather earlier.

\section{Hospital Vacuum Cleaner as Subject of Laboratory Study}

J. G. BATE (Princess Beatrice Hospital, London) said that to dispel uncertainty about the safety of suction cleaners in hospitals wards, the bacterial content of the air from 10 vacuum cleaners and three suction polishers was investigated. Bacteria-carrying particles were counted by means of a slit-sampler operating inside a large polythene reservoir in which stationary samples of exhaust air were collected from a polythene wind-tunnel in which the cleaners were operated. Serial samples of $5 \mathrm{cu}$. ft. were collected from machines before and after a 30-min. continuous run, during which the airflow was regularly interrupted every $15 \mathrm{sec}$. in order to stress the filters.

With one exception the vacuum cleaners gave colony counts not exceeding two in $10 \mathrm{cu}$. $\mathrm{ft}$., and retained not less than $97 \%$ of the number of airborne bacteria found in the room air immediately before the test. One machine consistently gave counts of up to five colonies, retaining only $90 \%$ of the aspirated organisms. Suction polishers gave counts up to 10 colonies. with retention between $90 \%$ and $94 \%$.

The aerial disturbance caused by vacuum-cleaner exhausts was also investigated; slit-sampler counts following the discharge of exhaust inside a small room showed that with open jet exhausts there was an immediate rise and fall in the number of airborne bacteria. followed by a slow bacterial fall-out over the next hour. When the speed of the airstream was reduced by means of a diffusing device at the outlet, no sign of disturbance was detected.

\section{Silicosis}

J. Gough (Cardiff) said that the latest theory of silicosis was the immunological one. Webster (1954) introduced it. A good summary of the present position is given by Vigliani and Pernis (1959). Pernis and Pecchiai (1954) showed that silicotic nodules contain $60 \%$ globulins including $\beta$ and $\gamma$ globulins. These occur as amorphous material in silicotic nodules. Electron microscopy shows collagen fibres embedded in the amorphous substance, the structure thus differing from simple scar tissue. Antweiler and Hirsch (1957) found that quartz coated with homologous serum albumin leads to the formation of antibodies when injected into laboratory animals. These antibodies can be detected by agglutination tests. Injected quartz acts as an adjuvant to the formation of antibodies in unrelated immunological reactions, e.g., the resistance to typhimurium infection is increased in mice prevously injected with tridymite (Pernis and Bolis, 1958). The relationship of an unrelated immunological reaction to silicosis was studied by Powell and Gough (1959), who showed that the immunization of rabbits with horse serum resulted in subsequently produced silicotic lesions being larger and more clearly demarcated and more collagenous. The anti-egg-albumen production in rabbits previously injected with tridymite was five to six times higher than in control rabbits (Ghiringhelli and Pernis, 1958). These observations are pertinent to the observation of Caplan (1953) that in rheumatoid miners the lung lesions are radiologically different from those of nonarthritic miners. Gough, Rivers, and Seal (1955) identified a corresponding difference in the pathology of the lesions, showing that pneumoconiosis renders the lung vulnerable to the nodular rheumatoid process. This may be an adjuvant immunological effect.

\section{REFERENCES}

Antweiler, H., and Hirsch, E. (1957). Z. In mun.-Forsch., 114, 378 Caplan, A. (1953). Thorax, 8, 29.

Ghiringhelli, L., and Pernis, B. (1958). Med. d. Lavoro, 49, 665.

Gough, J., Rivers, D., and Seal, R. M. E. (1955). Thorax, $10,9$.

Pernis, B., and Bolis, L. (1958). Proc. III Int. Symposium of Reticulo-endothelial Soc., Rapallo. (In the press.)

Powell, D. E. B., and Gough, J. (1959). Brit. J. exp. Path., 40, 40.

Vigliani, E. C., and Pernis, B. (1959). J. occup. Med., 1, 319.

Webster, I. (1954). Proc. Transvaal Mine Medical Officers' Ass., 34, 33 .

\section{Pneumoconiosis and Rheumatoid Disease}

ANTHONY CAPLAN (Cardiff) said that when a workman exposed to a dust hazard suffers from rheumatoid disease, he may develop a specific type of lung lesion. The lesions are nodular, about $0.5-1 \mathrm{~cm}$. in diameter, and distinguishable histologically from P.M.F. and classical silicosis. The lesions tend to conglomerate, and undergo liquefaction necrosis, fibrosis, and calcification.

The radiological appearances are characteristic and recognizable. Suddenly multiple, well-defined, round opacities, $0.5-5 \mathrm{~cm}$. in diameter, appear, distributed throughout both lung fields. The background of simple pneumoconiosis is often slight or absent. The opacities usually increase in size and number, and crops of fresh lesions may appear at intervals of a few months. Cavitation is common in the active phase. and calcification may occur in older lesions.

Most frequently the development of opacities coincides approximately with the onset of arthritis ; in a few cases the opacities precede the arthritis or the arthritis precedes the opacities by as much as six to 10 years. There is no relationship between the severity of arthritis and the extent and type of the radiological picture.

Epidemiological studies have shown that neither exposure to dust nor complicated pneumoconiosis is of any importance in the aetiology of rheumatoid arthritis.

The symptomatology is similar to that found in massive fibrosis, although there is often a surprising 
absence of constitutional symptoms-even when extensive cavitation is present-and only slight impairment of lung function even in the presence of widespread opacities.

Antituberculous chemotherapy and/or corticosteroid therapy have failed to affect the progression of the lung lesions.

The aetiology of the lesions remains obscure. The reaction of the lung to the presence of even minimal deposits of dust or dust and tuberculosis is obviously influenced by a rheumatoid factor. This factor has known agglutination properties. It is, therefore, possible that rheumatoid pneumoconiotic lesions may be due to an abnormal immunological process.

\section{Medico-legal Aspects of Pneumoconiosis}

W. R. L. JAmEs (Cardiff) referred to parts of the relevant acts and statutory instruments, including the Workmen's Compensation Acts, the Industrial Injuries Act, the Pneumoconiosis and Byssinosis Benefit Scheme, and the coroners' acts and rules.

Reference was made to the appropriate procedure for a doctor who suspected that his patient had died from pneumoconiosis.

The coroner's functions in pneumoconiosis cases were described, including his approach to the pathologist, his custody of tissues, and his possible procedures after receiving the pathologist's report.

The functions of the pathologist in pneumoconiosis cases were described, with his duties and privileges at the necropsy. Mention was made to the problem of the disposal of the body and how the pathologist could facilitate the necessary formalities.

Specific problems in assessing the cause of death were discussed, and the relationship of pneumoconiosis to some common natural diseases was indicated.

The constitution and functions of the pneumoconiosis medical panels were described, with emphasis on their duty to assess entitlement to death benefit.

\section{Pathology of the Lungs in Beryllium and Nickel Workers}

W. Jones Williams (Mount Vernon Hospital and Radium Institute, Northwood, Middlesex). Beryllium is increasingly used in industry, including atomic energy production. The inhalation of beryllium salts may lead to an acute or chronic disease, with an overall mortality of $27 \%$. The cardinal features are pulmonary, but it is a generalized disease.

Seven acute cases were studied, and the changes were non-specific tracheo-bronchitis and pneumonitis. Most patients recover completely and only $2 \%$ progress to the chronic form.

Chronic beryllium disease usually occurs without an antecedent acute phase. In a study of 52 chronic cases the histological lesion was found a noncaseating granuloma indistinguishable from Boeck's sarcoid and with identical inclusion bodies. Chronic beryllium disease leads to diffuse interstitial pulmonary fibrosis and death may ensue from cor pulmonale. Beryllium is not yet shown to be carcinogenic in man but is so in experimental animals.
Nickel refiners using the Mond process have an increased incidence of lung cancer. There is no recognized pneumoconiosis but some degree of interstitial fibrosis was found in five cases examined. Two showed "honeycomb" lung and four had pulmonary carcinoma together with recent bronchopneumonia. There was a minimal amount of carbonaceous dust and no evidence of silicosis. The diffuse fibrosis could be post-inflammatory but may be due to inhalation of mixed industrial dust, including metallic nickel. Two cases were analysed and showed 90 and 120 times the amount of nickel found in controls. The gas nickel carbonyl, an intermediary compound in the Mond process, is carcinogenic to laboratory animals.

\section{"Cadmium Pneumoconiosis"}

J. P. Smith (Manchester) described how cadmium had been used extensively in industry during the past 30 years. Inhalation hazards arise from exposure to cadmium oxide dust or to cadmium fume liberated when the metal is heated and may be acute or chronic in onset. The fumes are odourless and have no immediate irritant effect and can be inhaled in fatal concentration without anyone being aware of the danger.

Acute poisoning develops a few hours after exposure to high concentrations of the fume and leads to dyspnoea and pulmonary oedema. Striking reactive changes are seen in the alveolar epithelium of fatal cases.

Chronic poisoning is insidious and follows repeated exposure over several years to moderate concentrations of fume or oxide dust. Emphysema develops which, in severe cases, progresses to respiratory failure despite removal from exposure.

\section{Pulmonary Fibrosis after Exposure to Aluminium Powder}

G. B. ManNing (Bolton) reported that in 1954 a man aged 22 years died from pulmonary fibrosis. He had begun to work in an atmosphere heavily contaminated with aluminium powder three years and five months before his death. The man who took over the identical job vacated by the previous case also died from pulmonary fibrosis in 1959, four years and nine months after starting this work. A third case of fibrosis of lung from the same works was first suspected from the radiological appearances of the chest before his place of employment was known. This patient had, in fact, only spent one year in that same factory (Mitchell, 1959).

Further investigations have shown that to date out of 20 men who were exposed to heavy concentrations of aluminium powder in the atmosphere for more than six months, two have died from pulmonary fibrosis, four have evidence of pulmonary fibrosis, 11 have normal chests, and three have refused examination. These investigations are being prepared for publication.

\section{REFERENCE}

Mitchell, J. (1959). Brit. J. industr. Med., 16, 123. 


\section{Farmer's Lung}

Roger M. E. Seal (Sully) said that the account of the disease was based mainly on cases from the west Wales area, when 41 new patients were seen in 1959 after the wet summer of 1958 .

An acute form following a single heavy exposure, as after threshing, with dyspnoea and dry cough with a fine "spotting" radiologically, was described. This type apparently resolved in three to six weeks without treatment.

A progressive type, which followed repeated exposure, was described as characterized by severe exertional dyspnoea, a dry cough, and radiologically a diffuse opacity of the bases with a background of miliary mottling. Laboratory investigation revealed a hypergamma globulinaemia and diffusional impairment of oxygen exchange across the alveolar capillary membrane with a reduced $\mathrm{pO}_{2}$ and $\mathrm{pCO}_{2}$.

The histology of the acute and progressive types consisted of thickening of alveolar septa with small round-cell infiltration and with superadded epithelioid foci mimicking sarcoidosis.

Examples of a chronic end-result of farmer's lung were described which clinically and radiologically presented as diffuse fibrosis and honeycomb and cystic lung. The histology also was that of severe pulmonary destruction with fibrosis and collagen-lined cystic areas.

The aetiology was discussed and the condition was differentiated from sarcoidosis and pulmonary moniliasis and aspergillosis. The mycology and bacteriology of good and mouldy hay were contrasted and it was considered that the disease represented a hypersensitivity reaction diffusely in the lung to one or more antigens in mouldy hay.

\section{Further Studies of Anaemia in Pregnancy}

C. GiLes (Stoke-on-Trent) reported that in a controlled trial of prophylactic folic acid during the last trimester of pregnancy, 758 women who received oral folic acid and iron showed significantly higher haemoglobin levels at term than 721 women treated with iron alone. Megaloblastic anaemia developed in 20 cases of the control group but in none of the patients treated with folic acid. This trial supports the concept that folic acid depletion tends to occur in late pregnancy, even in the absence of megaloblasts in the marrow.

Analysis of 156 cases of megaloblastic pregnancy anaemia, diagnosed over a period of three years, suggests that an inadequate diet and intestinal malabsorption are important factors predisposing to megaloblastic anaemia. Abnormally high faecal fat excretion was found in 12 out of 41 cases which were investigated, but in none of 17 normal pregnant women.

When megaloblastic anaemias of pregnancy were classified according to the types and relative numbers of megaloblasts in the marrow, an abnormal blood group distribution, with a significant increase of blood group $\mathbf{A}$, was found in cases with the more advanced degrees of megaloblastosis. These findings suggest that folic acid deficiency tends to occur in a large number of women in late pregnancy, but only in a certain group, with an inherited predisposition, will folic acid depletion result in florid megaloblastic anaemia.

\section{Direct and Indirect Precipitin Tests in Thyroid Disease}

J. M. MoORE (Stobhill Hospital, Glasgow) described a serum precipitin test. This, giving a positive result with thyroglobulin, is generally considered to indicate major auto-immune thyroiditis. However, in a substantial proportion of patients with this disease the precipitin test is negative.

Two methods, neither of them original, were used to render this type of test more sensitive: (1) Fourfold concentration of serum: three sera, out of 20 tested, gave a definite positive with concentrate when the result with neat serum was negative or doubtful. (2) The use of adjuvant sera. Sera from cases of auto-immune thyroiditis giving a band of clearing on Ouchterlony plates instead of a band of precipitate have already been described. Two such sera were used to test precipitin-negative sera for the presence of a component which would convert the clear band to a precipitin band, either by diffusion of test and adjuvant sera towards one another on Ouchterlony plates or by mixtures.

Twenty-nine per cent. (15) of sera-precipitin-negative by routine, or direct, test-from a series of cases of Hashimoto's disease and primary myxoedema gave positive results with the indirect test. The indirect test was positive in some cases with a weak direct precipitin but not in any with a strong direct precipitin. Eighty-eight direct-negative sera from patients without thyroid disease and 33 from patients with thyroid disease not of immune type gave negative indirect results. Four negative direct and positive indirect results were obtained in women with thyroid C.F. antibody in their serum but without clinically-apparent thyroid disease.

\section{First Experiences with the Autoanalyser}

M. Lubran (West Middlesex Hospital). Micromethods for urea and glucose employing, respectively, a 1 in 10 and 1 in 20 dilution of blood may be run satisfactorily at 40 an hour, if a water wash is incorporated between samples. This gives an effective running rate of 20 an hour and prevents a low-value sample being contaminated by a preceding high one. The alkaline phosphatase procedure is very satisfactory, but the acid phosphatase method recommended for the apparatus measures only total acid phosphatase. Formaldehyde-stable acid phosphatase can be measured by including formaldehyde in the buffer substrate. However, this sometimes gives rise to precipitation of protein and the recorder must be carefully watched to notice when this happens.

During the average working day, the " autoanalyser" saves the time of at least one trained technician and gives, consistently, a higher standard of accuracy than 
the technicians. As no glassware is used, the running costs are less than the labour costs involved in manual methods. Most emergency work can be fitted in to the machine's programme, but a small number of manual determinations will have to be carried out for true emergencies and for night work.

The "autoanalyser" is suitable not only for large laboratories but for smaller laboratories, where five or six different determinations, involving about eight samples each, may be carried out by one technician in a working day.

\section{The Application and Implications of Bone Marrow Grafting in Man}

J. G. Humble (Westminster Hospital Medical School, London University). Modern therapy, especially modern chemotherapy for malignant disease, tends to destroy the bone marrow and lymphatic system. As bone marrow suspensions will re-seed the aplastic marrow produced by irradiation in laboratory animals, the application of this method has been studied in man. Methods of obtaining bone marrow by puncture, of storing it at $-79^{\circ} \mathrm{C}$, and of administering it safely to the recipient have been worked out. In six of eight patients treated by whole chest irradiation marrow function was restored to the sternum by stored autologous marrow. In four out of nine cases treated by massive chemotherapy marrow function was similarly restored. The other patients died before marrow function could be restored. Some evidence of the effect of the treatment on the tumours was noted. The danger of infection. especially of moniliasis, was emphasized. Despite recent criticism, the results suggest that a true re-seeding of the marrow spaces takes place. Using homologous transplants, three cases of aplastic anaemia and five of acute leukaemia have been treated. No remissions have been obtained although the last leukaemic child treated was showing haematological recovery when overwhelming moniliasis supervened. It was emphasized that the use of stored autologous marrow permitted a much greater margin of safety in the vigorous treatment of malignant disease processes.

\section{Transplantation of Foetal Haemopoietic Cells}

H. E. M. KAY (Royal Marsden Hospital, London). The advantages and disadvantages of using foetal haemopoietic cells in marrow replacement therapy were discussed with particular reference to the problem of tolerance by the graft towards the host tissues.

Treatment of cases of leukaemia, of chemicallyinduced and idiopathic bone-marrow aplasia, and of agammaglobulinaemia was described and the reasons for success or failure analysed.

Finally the problems of foetal cell embolism and the use of foetus-to-foetus transplants were considered.

On the Rationale of Bone Marrow Grafting

L. G. LaJtha (Radiobiology Laboratory, Churchill Hospital, Oxford) said that, considering the popula- tion kinetics of the bone marrow in respect of regeneration, and the available $x$-ray dose response curves of mammalian cells (in respect of reproductive integrity) it appears that isologous marrow grafting only saves an equivalent time for one complete mitotic wave in the regeneration of the integer stem cells. Available experimental as well as theoretical evidence suggests that a similar time-saving might be achieved by simpler methods, i.e., venesection and/or T.A.B. injections.

The efficiency of shielded marrow in cases of partial body irradiation was stressed. It is suggested that, in man, marrow grafting is a procedure which could be avoided by simple substitution therapy and stem cell stimulation.

\section{Intestinal Biopsy in Coeliac Disease}

A. H. CAMERon (Birmingham) said that the histological findings in duodenal or jejunal biopsies from 37 children were described. The ages ranged from 3 months to 14 years. Using a technique for estimating the relative surface area of the mucosa, the biopsies were separated into two groups. The first showed no significant loss of mucosal surface area. They were obtained from 11 patients, one of whom was a case of coeliac disease who had responded well to a gluten-free diet. The other 10 showed no clinical evidence of coeliac disease. Of the remaining 26 , the clinical diagnosis was coeliac disease in 21 , probable coeliac disease in four, and uncertain in one. Only one was on a gluten-free diet at the time of biopsy. This group was subdivided according to the severity of the histological appearances. The histological grading may be of value in evaluating response to therapy. It was concluded that biopsy is a safe and important method of investigation in steatorrhoea of childhood.

\section{Coagulation Tests on Capillary Blood}

Katharine M. Dormandy and R. M. Hardisty (The Hospital for Sick Children, Great Ormond Street, London) presented a scheme for the routine investigation of coagulation disorders using free-flowing capillary blood. Blood, $0.2 \mathrm{ml}$., is taken into a buffered citrate-saline mixture and used for the "prothrombin and proconvertin" test of Owren and Aas (1951) and and thromboplastin screening test of Hicks and Pitney (1957); the clotting time of the capillary blood (Dale and Laidlaw, 1911-12) is also determined. These three tests are used as screening procedures; accurate identification of any defects revealed can be achieved by assay procedures carried out on the same specimen of blood.

Details of the methods were given, and examples of results obtained in various coagulation disorders shown. The sensitivity and accuracy of the tests were indicated by comparison with the results of tests performed on venous blood obtained simultaneously.

\section{REFERENCES}

Dale, H. H., and Laidlaw, P. P. (1911-12). J. Path. Bact., 16, 351. Hicks, N. D., and Pitney, W. R. (1957). Brit. J. Haemat., 3, 227. Owren, P. A., and Aas, K. (1951). Scand. J. clin. Lab. Invest., 3, 201. 